CYSTIC FIBROSIS

\title{
Burkholderia pseudomallei: another emerging pathogen in cystic fibrosis
}

\author{
M R O'Carroll, T J Kidd, C Coulter, H V Smith, B R Rose, C Harbour, S C Bell
}

Thorax 2003;58:1087-1091

See end of article for authors' affiliations

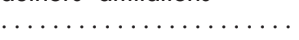

Correspondence to:

Dr S C Bell, Department of

Thoracic Medicine, The

Prince Charles Hospital

Rode Road, Chermside

4032, Australia:

scott_bell@health.qld.

gov.au

Received

28 December 2002

Accepted 17 August 2003
Background: Burkholderia pseudomallei is an important cause of acute fulminant pneumonia and septicaemia in tropical regions of northern Australia and south east Asia. Subacute and chronic forms of the disease also occur. There have been three recent reports of adults with cystic fibrosis (CF) who presumably acquired $B$ pseudomallei infection during extended vacations or residence in either Thailand or northern Australia.

Methods: The clinical course, molecular characteristics, serology and response to treatment are described in four adult CF patients infected with $B$ pseudomallei. Polymerase chain reaction (PCR) based methods were used to confirm $B$ pseudomallei and exclude $B$ cepacia complex. Genotyping was performed using randomly amplified polymorphic DNA (RAPD) PCR and pulsed field gel electrophoresis (PFGE).

Results: Four patients are described with a mean duration of infection of 32 months. All but one patient lived in tropical Queensland. Two patients (with the longest duration of infection) deteriorated clinically and one subsequently died of respiratory failure. Both responded to intravenous treatment specifically targeting $B$ pseudomallei. Another patient suffered two severe episodes of acute bronchopneumonia following acquisition of $B$ pseudomallei. Eradication of the organism was not possible in any of the cases. PFGE of a sample isolate from each patient revealed the strains to be unique and RAPD analysis showed retention of the same strain within an individual over time.

Conclusions: These findings support a potential pathogenic role for B pseudomallei in CF lung disease, producing both chronic infection and possibly acute bronchopneumonia. Identical isolates are retained over time and are unique, consistent with likely environmental acquisition and not person to person spread. B pseudomallei is emerging as a significant pathogen for patients with CF residing and holidaying in the tropics.
B urkholderia pseudomallei, the causative organism of melioidosis, is an endemic Gram negative bacterium found in soil and water in northern Australia and south east Asia. Sporadic cases have been reported elsewhere. Melioidosis is an important cause of acute fulminant pneumonia and septicaemia in endemic areas. Subacute and chronic forms of the disease also occur, albeit more commonly in non-endemic regions, and $B$ pseudomallei is capable of causing pyogenic or granulomatous infection in virtually any tissue. Infection in most cases is presumably acquired either by direct inoculation, inhalation or ingestion, and the nature of the host response determines the outcome of infection. This can range from asymptomatic seroconversion to fulminant sepsis and death. In a recent study of 252 cases of melioidosis in northern Australia, Currie et al reported that $80 \%$ of affected individuals had an identifiable risk factor predisposing to infection ${ }^{1} ; 27 \%$ of cases had chronic lung disease, a risk factor not previously identified, and $85 \%$ presented between December and February which is classed as the wet (cyclone) season in tropical Australia.

Four reports of $B$ pseudomallei infection in subjects with cystic fibrosis (CF) have recently been published..$^{2-5}$ The first two single case reports involved travellers visiting Thailand from Europe and both had pulmonary exacerbations soon after presumed acquisition. ${ }^{2}{ }^{3}$ There was a significant delay (6 years in one case) in identification of the organism. Both patients had chronic Pseudomonas aeruginosa and Burkholderia cepacia infection before travel and subsequently had chronic infection with $B$ pseudomallei. Eradication of B pseudomallei was achieved in one patient following 36 weeks of continuous antibiotic treatment. A third case of melioidosis in a patient with CF was reported in a research letter describing imported melioidosis in England and Wales, but clinical details were limited. ${ }^{4}$ Most recently, a case series from New Zealand described four CF patients (three children and one adult) who acquired $B$ pseudomallei infection after exposure in northern Australia, a region of endemnicity. ${ }^{5}$ Two of these patients suffered clinical deterioration following acquisition, one had asymptomatic carriage, and one underwent successful eradication. B pseudomallei may therefore be an emerging problem in CF and represent an important pathogen, not only in travellers to endemic regions but also to those patients with CF living in tropical climates.

The Adult CF Unit at The Prince Charles Hospital in Brisbane provides care to the majority of adults with CF in the state of Queensland (latitude range $10^{\circ}-28^{\circ}$ south), the northern part of which is tropical and endemic for $B$ pseudomallei. We describe four cases of $B$ pseudomallei infection, three of whom live in the tropics. We also present the molecular evidence for identification of B pseudomallei and longitudinal genotyping of strain types.

\section{METHODS}

\section{Laboratory identification}

Microbiology

All isolates were obtained from sputum that was collected and routinely cultured on the following media: Maconkey agar No 2 (bioMérieux Vitek Inc, Hazelwood, MO, USA), sheep blood agar (bioMérieux Vitek Inc), chocolate agar + bacitracin (Oxoid Ltd, Basingstoke, UK), cepacia agar (Mast 
Group Ltd, Merseyside, UK), and mannitol salt agar (Oxoid Ltd). Phenotypic identification of B pseudomallei was based on biochemical reactions, a positive oxidase test, and the API 20NE. Isolates were stored in liquid nitrogen using PROTECT bacterial preservers (Technical Service Consultants Ltd, Heywood, UK).

\section{Antibiotic sensitivity}

Antibiotic sensitivity testing was conducted according to the National Committee for Clinical Laboratory Standards (NCCLS). ${ }^{6}$ Disc diffusion was employed using Mueller Hinton agar and Oxoid antibiotic discs (Oxoid Ltd). The following antibiotics (disc concentration) were tested: tetracycline $(30 \mu \mathrm{g} / \mathrm{ml})$, gentamicin $(10 \mu \mathrm{g} / \mathrm{ml})$, sulphamethoxazole/trimethoprim (SXT) $(25 \mu \mathrm{g} / \mathrm{ml})$, tobramycin $(10 \mu \mathrm{g} /$ $\mathrm{ml})$, amikacin $(30 \mu \mathrm{g} / \mathrm{ml})$, ceftazidime $(30 \mu \mathrm{g} / \mathrm{ml})$, piperacillin $(100 \mu \mathrm{g} / \mathrm{ml})$, ticarcillin/clavulanic acid $(75 / 10 \mu \mathrm{g} / \mathrm{ml})$, ciprofloxacin $(5 \mu \mathrm{g} / \mathrm{ml})$, aztreonam $(30 \mu \mathrm{g} / \mathrm{ml})$, meropenem $(10 \mu \mathrm{g} / \mathrm{ml})$, imipenem $(10 \mu \mathrm{g} / \mathrm{ml})$, and colistin sulphate $(10 \mu \mathrm{g} / \mathrm{ml})$. SXT sensitivities were confirmed using E tests according to NCCLS broth dilution break points.

\section{Multiple combination bactericidal testing (MCBT)}

MCBT was carried out to identify bactericidal or antagonistic combinations of antibiotics. The procedure was performed in triplicate in 96 well round bottomed microtitre plates (Nunc Inc, Roskilde, Denmark) as described by Aaron et al. ${ }^{7}$ The following antibiotics were used: tobramycin (Sigma Chemicals) $10 \mu \mathrm{g} / \mathrm{ml}$ and $200 \mu \mathrm{g} / \mathrm{ml}$ (the latter suitable for nebulised use), meropenem (Astra Zeneca) $32 \mu \mathrm{g} / \mathrm{ml}$, amikacin and cefepime (Bristol-Myers Squibb) both $32 \mu \mathrm{g} / \mathrm{ml}$, ceftazidime and ticarcillin/clavulanic acid (Glaxo Smith Kline) $32 \mu \mathrm{g} / \mathrm{ml}$ and $32 / 2 \mu \mathrm{g} / \mathrm{ml}$ respectively, ciprofloxacin (Bayer) $2 \mu \mathrm{g} / \mathrm{ml}$, chloramphenicol, SXT and doxycycline (all Roche Laboratories, Canada) $20 \mu \mathrm{g} / \mathrm{ml}, 10 / 2 \mu \mathrm{g} / \mathrm{ml}$ and $4 \mu \mathrm{g} /$ $\mathrm{ml}$, respectively. Reference strains ( $P$ aeruginosa ATCC 27853 , $E$ coli ATCC 25922, and E coli ATCC 35218) were used to control for the efficacy of the antibiotics.

\section{Serological tests}

$B$ pseudomallei antibodies were measured in each patient using serum collected after the first identification of $B$ pseudomallei in sputum. Stored serum was available for retrospective testing in one case. Total antibody titre was determined using an indirect haemagglutination assay and $\operatorname{Ig} M$ and IgG subclasses were measured using an enzyme linked immunoassay. ${ }^{8}$

\section{Molecular identification 16 S rRNA polymerase chain reaction (PCR) for $B$ pseudomallei}

Isolates from each patient were confirmed as B pseudomallei using a modification of the method described by Dharakul et al. ${ }^{10}$ The primers used were Bps16S-42L (sense) and Bps16S-266R (antisense) or Bps16S-42L (sense) and Bpsl6S-427R (antisense) (two separate PCR reactions). DNA amplification was performed in an AB2700 Thermal Cycler (Applied Biosystems, Foster City, USA) with an initial hot start step of $95^{\circ} \mathrm{C}$ for 15 minutes, followed by 35 cycles of 1 minute at $95^{\circ} \mathrm{C}, 1$ minute at $60^{\circ} \mathrm{C}, 1$ minute at $72^{\circ} \mathrm{C}$, and final extension for 10 minutes at $72^{\circ} \mathrm{C}$. The presence of amplified DNA product was detected in $1.5 \%(\mathrm{w} / \mathrm{v})$ agarose gel stained with $2.5 \mu \mathrm{l} 10 \mathrm{mg} / \mathrm{ml}$ ethidium bromide. Bands at $243 \mathrm{bp}$ and $405 \mathrm{bp}$ were interpreted as being consistent with PCR amplification of arabinose negative $\left(\mathrm{Ara}^{-}\right)$B pseudomallei DNA (environmental $\mathrm{Ara}^{+}$isolates of $B$ pseudomallei are seldom encountered in clinical specimens and exhibit minimal virulence in animal models ${ }^{11}{ }^{12}$ ).
recA PCR for $B$ cepacia complex

$B$ cepacia was excluded using PCR amplification of the recA gene based on methods previously described. ${ }^{13}$ DNA extracted from each isolate was used as a template.

\section{RFLP analysis of $16 \mathrm{~S}$ rDNA}

The 16S rDNA-RFLP method as described by Segonds et al ${ }^{14}$ was performed on each isolate and the results compared with a known strain of $B$ pseudomallei. This method has been shown to discriminate effectively between members of the $B$ cepacia complex and other Burkholderia species. Restriction endonuclease digestion was performed on each PCR product separately using the enzymes DdeI and MspI. Each individual RFLP profile was assigned an identifying uppercase letter.

\section{Genotyping}

Genotyping was performed on available isolates from each patient collected over at least several months using randomly amplified polymorphic DNA (RAPD) and pulsed field gel electrophoresis (PFGE). RAPD analysis was performed using a modification of the method previously described by Corney et al. ${ }^{15}$ Strains were considered identical if their banding patterns were identical for all five primers. PFGE was performed according to the method of Currie et al using SpeI restriction endonuclease with the modification of adding thiourea to the running buffer. ${ }^{16}{ }^{17}$ The results were visually compared.

Clinical and microbiological review of patients attending The Prince Charles Hospital has been approved by the human research and ethics committee as part of the provision of patient data for the Australia and New Zealand Cystic Fibrosis Data Registry.

\section{RESULTS \\ Clinical description \\ Case 1}

A 23 year old man with $\mathrm{CF}(\Delta \mathrm{F} 508 / \Delta \mathrm{F} 508)$ living in tropical Queensland had $B$ pseudomallei initially identified in his sputum in May 2000. He had severe bronchiectasis (forced expiratory volume in 1 second $\left(\mathrm{FEV}_{1}\right) 40-45 \%$ predicted), CF related diabetes requiring insulin, and chronic $P$ aeruginosa and MRSA infection. Before identification of $B$ pseudomallei he required two to four admissions per year for parenteral antibiotic treatment. Shortly after the acquisition of $B$ pseudomallei he deteriorated significantly with increasingly frequent pulmonary exacerbations, which were characterised by marked constitutional symptoms and increased circulating inflammatory markers. His $\mathrm{FEV}_{1}$ fell from $40 \%$ predicted to $25 \%$ predicted over an 18 month period and he developed hypercapnic respiratory failure requiring acute non-invasive ventilatory support during one admission. The patient's clinical course appeared to stabilise in February 2002 when long term treatment with high dose SXT was commenced (800/160 mg twice daily). He subsequently deteriorated further despite the SXT and then stabilised again with the addition of amoxycillin/clavulanic acid (875/125 mg twice daily). He was listed for lung transplantation from March 2002 but progressive bronchial sepsis occurred and the patient died in November 2002 as a result of respiratory failure.

Despite several attempts at eradication, B pseudomallei continued to be isolated from the sputum (moderate to heavy growth). MRSA and $P$ aeruginosa were also usually but not invariably identified in the sputum and Stenotrophomonas maltophilia had been cultured infrequently. Parenteral antibiotics to attempt eradication of $B$ pseudomallei have included ceftazidime (up to $15 \mathrm{~g} /$ day) and meropenem (up to $6 \mathrm{~g} /$ day) for as long as 8 weeks. These have been used in combination with high dose oral SXT. Oral tetracycline and ciprofloxacin 
have been used individually and in combination with SXT with little benefit.

\section{Case 2}

A 36 year old man with CF $(\Delta \mathrm{F} 508 / \Delta \mathrm{F} 508)$ living in tropical Queensland had B pseudomallei first identified in the sputum in 1994. Previously the patient had been well with mild bronchiectasis $\left(\mathrm{FEV}_{1} \quad 65-75 \%\right.$ predicted) and had only previously required parenteral antibiotics on two occasions. Ten years previously the patient's mother had been successfully treated for acute melioidosis (severe pneumonia). They shared accommodation before the episode of pneumonia and for several years after recovery, and she remains well. Subsequent to the identification of $B$ pseudomallei, he gradually deteriorated. The patient's $\mathrm{FEV}_{1}$ is currently $35 \%$ predicted. Pulmonary exacerbations have become increasingly frequent and are typically accompanied by systemic symptoms. On two occasions the patient received 2 weeks of high dose intravenous ceftazidime, meropenem and oral SXT which resulted in improved spirometry; however, B pseudomallei persisted in the sputum. The patient continues to require up to four courses of intravenous antibiotics per year, including either ceftazidime or meropenem and oral SXT, for pulmonary exacerbations with accompanying clinical response. Between exacerbations he is maintained on SXT or tetracycline. Most sputum samples have a pure light to heavy growth of $B$ pseudomallei. $P$ aeruginosa is also cultured infrequently.

\section{Case 3}

A 15 year old boy with CF $(\Delta \mathrm{F} 508 / \mathrm{G} 542 \mathrm{X})$ from tropical far north Queensland had $B$ pseudomallei first isolated in the sputum in December 2001. The patient has mild bronchiectasis with two previous admissions (aged 8 and 14 years) for parenteral treatment of pulmonary exacerbation. Before December 2001 Staphylococcus aureus and $P$ aeruginosa had been isolated in the sputum and $B$ pseudomallei has continued to be isolated from the sputum subsequently (scant to light growth). The patient has remained well and has not received any specific treatment targeting $B$ pseudomallei. The patient had an older sibling with CF who had chronic $B$ cenocepacia (genomovar IIIb) infection and died of respiratory failure in April 2001.

\section{Case 4}

A 24 year old woman with $\mathrm{CF}(\Delta \mathrm{F} 508 / \Delta \mathrm{F} 508)$, moderate bronchiectasis $\left(\mathrm{FEV}_{1} 65 \%\right.$ predicted), and chronic $B$ cenocepacia (genomovar IIIb) infection (Australian epidemic strain, LMG18830) relocated to subtropical Queensland from a temperate region in New South Wales in 1998. She typically had required four courses of parenteral antibiotic treatment for pulmonary exacerbations per year. Sputum intermittently cultured $P$ aeruginosa and $S$ aureus in addition to $B$ cepacia. In a separate analysis to determine whether $B$ pseudomallei antibodies were positive in CF patients infected with $B$ cepacia, we identified very high titres in this patient in April 2002. The patient had neither resided nor visited tropical regions within Australia or south east Asia. Subsequent analysis of stored serum from March 2000 and July 2001 also revealed positive $B$ pseudomallei antibodies suggesting exposure prior to 2000. Furthermore, there was an eightfold rise in antibody titre between March 2000 and July 2001. No other patient with $B$ cepacia treated in the unit (eight patients) had positive $B$ pseudomallei antibodies. Subsequent re-examination of sputum revealed $B$ pseudomallei in addition to $B$ cepacia. Sputum now consistently grows both $B$ pseudomallei (scant to heavy growth) and $B$ cepacia. In June 2001 the microbiology laboratory identified an organism from the sputum atypical for $B$ cepacia which had an antibiogram identical to the subsequently identified $B$ pseudomallei. The patient has had two severe exacerbations complicated by bronchopneumonia in July 2001 and June 2002, both of which responded to 3 week courses of intravenous ceftazidime, meropenem, and tobramycin.

\section{Laboratory identification \\ Microbiology}

Each of the isolates induced a pink colouration when grown on MAST $B$ cepacia medium. Colonies on the selective medium were similar to that of $B$ cepacia but could be distinguished by a slightly drier appearance. Older cultures ( $>3$ days) exhibited the characteristic wrinkled colonial morphology of this organism. A distinct "earthy" odour emanated from each of the isolates when cultured onto solid media. Each isolate was identified as B pseudomallei based on the API20NE with cases 1, 2 and 4 sharing the same profile (1156577, 99.9\% B pseudomallei). The API20NE profile for case 3 was 1556577 (99.8\% B pseudomallei). Co-pathogens included $P$ aeruginosa (three cases), $B$ cepacia (one patient), $S$ aureus (two patients), and MRSA (one patient). In two cases $B$ pseudomallei was the dominant pathogen cultured in the sputum based on it being the sole organism cultured in the majority of sputum samples (cases 1 and 2).

\section{Antibiotic sensitivity}

All isolates were resistant to all classes of aminoglycosides and colistin. All but one of the isolates was sensitive to ceftazidime and all were sensitive to the carbapenem antibiotics, meropenem and imipenem. Three of the four isolates were sensitive to tetracycline and SXT, but only one was sensitive to ciprofloxacin.

\section{MCBT}

All isolates were tested against 11 single, 47 double, and 34 triple antibiotic combinations. High dose tobramycin $(200 \mu \mathrm{g} / \mathrm{ml})$ and ticarcillin/clavulanic acid were the most effective single agents, being bactericidal for all four isolates, while meropenem, ceftazidime, and cefepime were bactericidal for three of the four isolates. High dose tobramycin in combination with all single antibiotics except chloramphenicol was bactericidal for all four isolates; high dose tobramycin with chloramphenicol was bactericidal for three of the four isolates. Among dual combinations suitable for intravenous or oral use, meropenem with SXT or tobramycin $(10 \mu \mathrm{g} / \mathrm{ml})$ in combination with meropenem or ceftazidime or cefepime were most effective being bactericidal for all four isolates. The addition of a third antibiotic to these low dose tobramycin combinations was counterproductive in two of the four cases. Some combinations including ceftazidime and meropenem were bactericidal for three of the isolates but antagonistic for the fourth isolate.

\section{Serological tests}

All patients had positive serum $B$ pseudomallei antibodies which, in three cases (1-3), were performed after the initial identification of $B$ pseudomallei in sputum. Case 4 had positive antibodies before the first laboratory identification of $B$ pseudomallei in sputum, with seroconversion prior to July 2001. All cases had positive $B$ pseudomallei total antibody and IgG, but only cases 1 and 4 had positive IgM antibodies.

\section{Molecular identification $16 S$ rRNA PCR for B pseudomallei}

The $B$ pseudomallei 16S rRNA PCR was performed on DNA extracted from each isolate, producing bands at $243 \mathrm{bp}$ and $405 \mathrm{bp}$ in each case, consistent with $\mathrm{Ara}^{-} \mathrm{B}$ pseudomallei. 


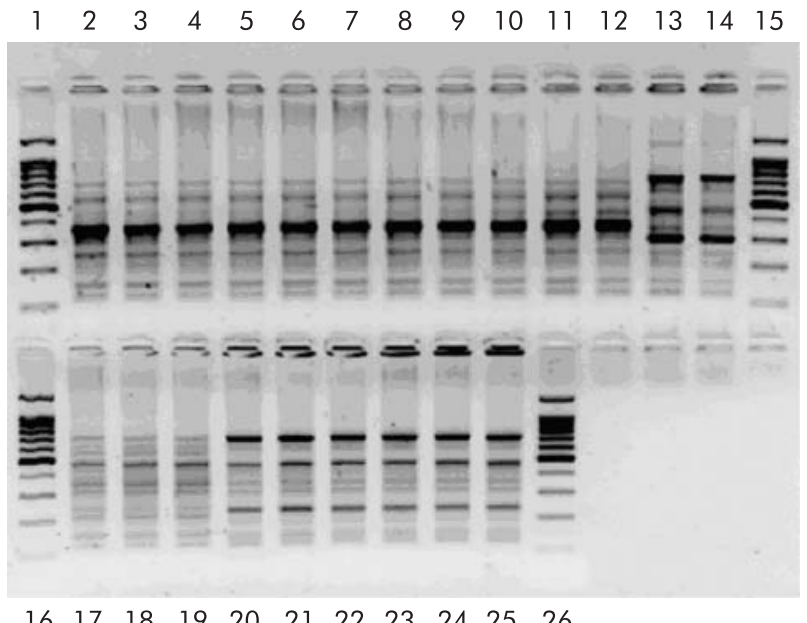

Figure 1 Comparison of the banding patterns from randomly amplified polymorphic DNA PCR assays of sequential $B$ pseudomallei isolates from each of the cases. The RAPD result shown is for the L10a primer set. Lanes $2-12$, case 1 ; lanes 13 and 14 , case 4 ; lanes 17-19, case 3; and lanes $20-25$, case 2 . Lanes $1,15,16$, and $26=100$ bp molecular weight ladder.

\section{recA PCR for B cepacia complex and RFLP analysis of 16S rDNA}

The recA PCR for B cepacia complex was negative for each isolate. RFLP profiles with DdeI and MspI yielded an AA pattern in each isolate which was identical to that obtained previously in our laboratory with $B$ cepacia (genomovar I), B cenocepacia (genomovar III), and B anthina (genomovar VIII).

\section{Genotyping}

Multiple isolates were available for genotypic analysis ranging from two to 11 per patient collected over 25 months from April 2000 to May 2002. PFGE revealed that a sample isolate from each patient was distinct and unrelated to other patients. RAPD analysis revealed retention of the same strain within an individual over time (fig 1).

\section{DISCUSSION}

Burkholderia pseudomallei is an important pathogen in people living in endemic areas of northern Australia and south east Asia. In a recent study from northern Australia chronic lung disease was recognised as a risk factor for melioidosis. ${ }^{1}$ We report a case series of $B$ pseudomallei infection in CF. Three of the four cases lived in endemic areas at the time of colonisation while the fourth had never visited tropical regions. Acquisition of melioidosis in temperate south eastern Queensland has previously been reported..$^{18}$ There have been four previous reports of $B$ pseudomallei infection in $\mathrm{CF}^{2-5}$ The first two were visitors to an endemic area with infection apparently acquired during vacation in south east Asia. ${ }^{23}$ Most recently, Holland et al described $B$ pseudomallei infection in four patients with $\mathrm{CF}$, three of whom were children who had previously resided in Darwin, northern Australia. ${ }^{5}$ Clinics and microbiology laboratories need to consider and be able to accurately identify $B$ pseudomallei in patients with CF residing within or returning from endemic regions.

The current report provides preliminary evidence that $B$ pseudomallei is a pathogen in the respiratory tract in patients with CF. B pseudomallei was the dominant organism in at least two of the patients and, in one of these, it was frequently the only organism cultured in the sputum. Both these patients steadily declined following acquisition and both had recurrent exacerbations characterised by constitutional symptoms and raised serum inflammatory markers. To date, case 3 has had no deleterious effects associated with $B$ pseudomallei infection. The fourth patient suffered two severe episodes of bronchopneumonia following the acquisition of B pseudomallei. During the first episode of bronchopneumonia in June 2001 an unidentified organism with an antibiogram consistent with $B$ pseudomallei was cultured from the sputum. Retrospective analysis of stored serum revealed an eightfold rise in the titre of melioid antibodies between March 2000 and July 2001. A specific immune response to B pseudomallei was also evident in each of the other cases. Taken together, these findings support a potential pathogenic role for $B$ pseudomallei in CF lung disease, producing both chronic infection and possibly acute bronchopneumonia.

Attempts to eradicate $B$ pseudomallei have so far been unsuccessful. The one previous report describing eradication in a patient with CF required 36 weeks of continuous antibiotic treatment. Prolonged courses of antibiotics specifically targeting $B$ pseudomallei in three of the four cases have been administered and eradication was not possible. The most effective antibiotics in terms of clinical response were ceftazidime and meropenem when used in combination. Most (but not all) of the patients had $B$ pseudomallei isolates that were sensitive to these antibiotics. Continuous high dose SXT resulted in stabilisation in case 1 which had proved difficult to achieve despite multiple courses of triple parenteral antibiotics. While the effectiveness of MCBT has not yet been validated in clinical trials, the results of this study suggest that it may have a role in the management of patients with $B$ pseudomallei. The combination of ceftazidime and meropenem, commonly used in the treatment of this organism and clinically useful in three of the four cases reported here, was also bactericidal by MCBT. MCBT may also provide new options for strategies to treat and potentially eradicate $B$ pseudomallei. Several combinations not usually associated with the treatment of this organism were found to be bactericidal for the majority of isolates. Among these were combinations involving tobramycin tested at the high concentration reported to be achievable in the airways following nebulisation. A recent Cochrane review of interventions for treating melioidosis recommended that regimens for the acute phase of the illness should contain ceftazidime or imipenem. ${ }^{19}$ The same review suggested that oral maintenance therapy after the acute phase should consist of a three drug regimen containing chloramphenicol, doxycycline, and SXT. Our clinical experience is consistent with these recommendations. Interestingly, combinations of chloramphenicol with doxycycline or doxycycline with SXT were not bactericidal for any of the isolates, but MCBT was not performed on the triple combination of chloramphenicol, doxycycline, and SXT.

Identification of $B$ pseudomallei in previously reported cases has proved difficult. ${ }^{23}$ Even in a specialist laboratory dealing with CF microbiology, $B$ pseudomallei can be mistaken for $B$ cepacia when using commercially available identification systems. ${ }^{20} \mathrm{~A}$ high index of suspicion is needed, not only in clinics that provide care for patients living in tropical areas but also in those clinics caring for patients travelling to endemic areas. Laboratories outside endemic areas should be aware of the various characteristics and identification procedures that may be used if $B$ pseudomallei is suspected. The organism can be readily grown on most respiratory solid culture media and is characteristically colistin resistant and therefore easily grown on $B$ cepacia medium. Although similar to $B$ cepacia complex, $B$ pseudomallei is a distinct species. Its colonies exhibit a slightly drier appearance and earthy odour, and older cultures have a characteristic wrinkled appearance. These subtle phenotypic differences underscore the need for appropriate molecular confirmation of $B$ pseudomallei and exclusion of $B$ cepacia complex. We have shown that 
$B$ pseudomallei produces an identical RFLP fingerprint to that of $B$ cepacia, $B$ cenocepacia, and $B$ anthina using $16 \mathrm{~S}$ rDNA RFLP analysis, which again highlights the importance of the two specific PCR assays to differentiate $B$ pseudomallei from $B$ cepacia complex. ${ }^{14}$

Most cases of infection with $B$ pseudomallei are presumed to occur via direct inoculation from contaminated soil or water. Four cases of person to person transmission have been described $^{21-23}$ and one of these involved two siblings with CF. ${ }^{5}$ Each of these four cases involved close contact with the index case, with either prolonged exposure or direct exposure to open wounds. None of the patients in our series had overlapping admissions or social contact. The B pseudomallei strain from each individual patient was genetically distinct using molecular techniques, suggesting environmental acquisition and not person to person spread. Furthermore, each of the patients retained identical $B$ pseudomallei strains over prolonged periods. The uniqueness of $B$ pseudomallei strains in our patients is consistent with the known epidemiology of this organism, with clonal outbreaks being unusual. ${ }^{16}$ None of the cases had excessive exposure to soil or water and, while case 2 had lived with his mother at the time that she contracted melioidosis, a decade had elapsed before he developed the first signs of $B$ pseudomallei infection. It therefore seems unlikely that case 2 acquired $B$ pseudomallei infection from his mother. The potential transmissibility of $B$ pseudomallei raises infection control issues. The cases of person to person transmission described to date have mostly involved prolonged close contact with patients harbouring identifiable risk factors for acquisition. ${ }^{51-23}$ Our approach has been to manage these patients in the same manner as those infected with $B$ cepacia. They attend clinic on a separate day, are managed in a separate ward as inpatients, and are advised to avoid close contact with other CF patients. Staff are asked to follow standard universal precautions and we have not imposed any other specific infection control measures. The evidence thus far would suggest that patients suffering from melioidosis do not pose a significant risk to otherwise well healthcare workers.

At a time when subjects with CF are undertaking international travel more than ever before, B pseudomallei is emerging as a significant pathogen for those holidaying in the tropics. A recent editorial has highlighted the importance of careful planning for air travel in patients with CF. ${ }^{24}$ Patients should be advised to avoid soil and exposure to fresh water supplies such as swimming lagoons when visiting endemic areas, particularly during the wet season. They should also be warned of the potential for infection to occur in the absence of identifiable exposure ${ }^{25}$ and that CF places them at an increased risk of acquisition. CF care providers need to have an awareness of $B$ pseudomallei and the potential risk it poses for the traveller with CF.

\section{ACKNOWLEDGEMENTS}

The authors thank Ms Monica Singh for performing the MCBT assays and Ms Leonie Barnett for the development of the RAPD assay.

The MCBT studies were performed with the financial support of Cystic Fibrosis Australia Research Trust.

\section{Authors' affiliations}

M R O'Carroll, S C Bell, Adult Cystic Fibrosis Unit, The Prince Charles Hospital, Brisbane, Queensland, Australia
T J Kidd, C Coulter, Queensland Health Pathology Service, The Prince Charles Hospital, Brisbane, Queensland, Australia

H V Smith, Public Health Microbiology, Queensland Health Scientific Services, Brisbane, Queensland, Australia

B R Rose, C Harbour, Department of Infectious Diseases and Immunology, University of Sydney, New South Wales, Australia

S C Bell, University of Queensland, Brisbane, Queensland, Australia

\section{REFERENCES}

1 Currie BJ, Fisher DA, Howard DM, et al. Endemic melioidosis in tropical northern Australia: a 10-year prospective study and review of the literature. Clin Infect Dis 2000;31:981-6.

2 Visca P, Cazzola G, Petrucca A, et al. Travel-associated Burkholderia pseudomallei infection (melioidosis) in a patient with cystic fibrosis: a case report. Clin Infect Dis 2001;32:e15-6.

3 Schulin T, Steinmetz I. Chronic melioidosis in a patient with cystic fibrosis. J Clin Microbiol 2001;39:1676-7.

4 Dance DAB, Smith MD, Aucken HM, et al. Imported melioidosis in England and Wales. Lancet 1999;353:208.

5 Holland DJ, Wesley A, Drinkovic, et al. Cystic fibrosis and Burkholderia pseudomallei infection: an emerging problem? Clin Infect Dis 2002;35:e138-40.

6 National Committee for Clinical Laboratory Standards. Performance standard for antimicrobial susceptibility testing Document M100-S10. Wayne, PA: National Committee for Clinical Laboratory Standards, 2002.

7 Aaron SD, Ferris W, Henry DA, et al. Multiple combination bactericidal antibiotic testing for patients with cystic fibrosis infected with Burkolderia cepacia. Am J Respir Crit Care Med 2000;161:1206-12.

8 Alexander AD, Huxsoll DL, Warner AR Jr, et al. Serological diagnosis of human melioidosis with indirect hemagglutination and complement fixation tests. Appl Microbiol 1970;20:825-33.

9 Ashdown LR. Indirect haemagglutination test for melioidosis. Med J Aust 1987; 147:364-5.

10 Dharakul T, Tassaneetrithep B, Trakulsomboon S, et al. Phylogenetic analysis of $\mathrm{Ara}^{+}$and $\mathrm{Ara}^{-}$Burkholderia pseudomallei isolates and development of a multiplex PCR procedure for rapid discrimination between the two biotypes. $J$ Clin Microbiol 1999;37:1906-12.

11 Smith MD, Angus BJ, Wuthiekanun V, et al. Arabinose assimilation defines a nonvirulent biotype of Burkholderia pseudomallei. Infect Immunol 1997:65:4319-21.

12 Trakulsomboon S, Vuddhakul V, Tharavichitkul P, et al. Epidemiology of arabinose assimilation in Burkholderia pseudomallei isolated from patients and soil in Thailand. Southeast Asian J Trop Med Public Health 1999;30:756-9.

13 Mahenthiralingam E, Bischof J, Byrne SK, et al. DNA-Based diagnostic approaches for identification of Burkholderia cepacia complex, Burkholderia vietnamiensis, Burkholderia multivorans, Burkholderia stabilis, and Burkholderia cepacia genomovars I and III. J Clin Microbiol 2000;38:3165-73.

14 Segonds C, Heulin T, Marty N, et al. Differentiation of Burkholderia species by PCR-restriction fragment length polymorphism analysis of the 16S rRNA gene and application to cystic fibrosis isolates. J Clin Microbiol 1999;37:2201-8.

15 Corney B, Colley J, Djordjevic S, et al. Rapid identification of some Leptospira isolates from cattle by random amplified polymorphic DNA fingerprinting. J Clin Microbiol 1993;31:2927-32.

16 Currie BJ, Mayo M, Anstey NM, et al. A cluster of melioidosis cases from an endemic region is clonal and is linked to the water supply using molecular typing of Burkholderia pseudomallei isolates. Am J Trop Med Hyg 2001;65:177-9.

17 Römling U, Tümmler B. Achieving 100\% typability of Pseudomonas aeruginosa by pulsed-field gel electrophoresis. J Clin Microbiol 2000;38:464-5.

18 Scott IA, Bell AM, Staines DR. Fatal human melioidosis in south-eastern Queensland. Med J Aust 1997;166:197-9.

19 Samuel M, Ti TY. Interventions for treating melioidosis (Cochrane Review). In: The Cochrane Library, Issue 2. Oxford: Update Publications, 2002.

20 Lowe P, Engler C, Norton R. Comparison of automated and nonautomated systems for identification of Burkholderia pseudomallei. J Clin Microbiol 2002;40:4625-7.

21 Abbink FC, Orendi JM, De Beaufort AJ. Mother-to-child transmission of Burkholderia pseudomallei. N Engl J Med 2001;344:1171.

22 Kunakorn M, Jayanetra P, Tanphaichitra D. Man-to-man transmission of melioidosis. Lancet 1991;337:1290-1.

23 McCormick JB, Sexton DJ, McMurray JG, et al. Human-to-human transmission of Pseudomonas pseudomallei. Ann Intern Med 1975;83:512-3.

24 Webb AK. Flying and cystic fibrosis: getting there and back safely. Thorax 2001;56:821-2.

25 Schwarzmaier A, Riezinger-Geppert F, Schober G, et al. Fulminant septic melioidosis after a vacation in Thailand. Wien Klin Wochenschr 2000;1 12:892-5. 